\title{
Carnosic acid potentiates the anticancer effect of temozolomide by inducing apoptosis and autophagy in glioma
}

\author{
Naiyuan Shao ${ }^{1,2} \cdot J_{i a h a o} \mathrm{Mao}^{3} \cdot \operatorname{Lian} \mathrm{Xue}^{1,3} \cdot$ Rong Wang $^{1,3} \cdot$ Feng Zhi ${ }^{1,3}$ (D) Qing Lan $^{2}$
}

Received: 29 July 2018 / Accepted: 1 November 2018 / Published online: 20 November 2018

(c) The Author(s) 2018, corrected publication 2022

\begin{abstract}
Objective Malignant glioma is a lethal brain tumor with a low survival rate and poor prognosis. New strategies are urgently needed to augment the chemotherapeutic effects of temozolomide (TMZ), the standard drug in glioma treatment. Carnosic acid (CA) has been reported to have anticancer, antioxidant and anti-infectious properties. In this study, we aimed to investigate the anticancer effects and the underlying mechanisms of CA in combination with TMZ in glioma cancer cells.

Methods The glioma cancer cells were treated with TMZ, CA, or TMZ + CA. We evaluated cell survival by CCK-8 assay, cell anchorage-independent survival by colony formation assay, cell migration by wound-healing assay, cell cycle and cell apoptosis by flow cytometry, and protein expression by western blot.

Results CA enhanced the cytotoxic effect of TMZ in glioma cancer cells. CA enhanced TMZ-induced inhibition of colony formation and cell migration and enhanced TMZ-induced cell cycle arrest and cellular apoptosis. Immunofluorescence suggested that CA in combination with TMZ triggered autophagy. Furthermore, CA promoted TMZ-induced cell cycle arrest and cellular apoptosis by Cyclin B1 inhibition and activation of PARP and Caspase-3, while CA promoted TMZ-induced cellular autophagy by p-AKT inhibition, p62 downregulation and LC3-I to LC3-II transition.

Conclusion These data suggest that the combination therapy of CA and TMZ strengthens the anticancer effect of TMZ by enhancing apoptosis and autophagy.
\end{abstract}

Keywords Carnosic acid $\cdot$ Temozolomide $\cdot$ Apoptosis $\cdot$ Autophagy $\cdot$ Glioma

\section{Introduction}

Glioma, which is the most frequent primary tumor in the brain, accounts for almost half of all brain tumors in the United States and in China [1]. According to the World

Naiyuan Shao and Jiahao Mao have contributed equally in this work.

Feng Zhi

danielzhif@suda.edu.cn

Qing Lan

qinglan_sz@126.com

1 Department of Neurosurgery, Third Affiliated Hospital of Soochow University, Changzhou, Jiangsu, China

2 Department of Neurosurgery, The Second Affiliated Hospital of Soochow University, \#1055 Sanxiang Road, Suzhou, Jiangsu, China

3 Modern Medical Research Center, The Third Affiliated Hospital of Soochow University, \#185 Juqian Road, Changzhou, Jiangsu, China
Health Organization (WHO) classification system, glioblastoma (GBM), the Grade IV glioma, is the most malignant glioma [2]. The current strategy for GBM is surgical resection followed by radiotherapy and adjuvant temozolomide (TMZ) chemotherapy [3]. Though significant improvement has been achieved in GBM therapeutic management, the patient 5-year survival rate is only 5.5\% [1]. TMZ, an oral alkylating agent, is the first-line chemotherapy agent for glioma [4]. Its cytotoxicity results from inducing tumor cell apoptosis, autophagy and the unfolded protein response by alkylating DNA at the guanine residues [5]. One of the main causes for treatment failure is TMZ chemoresistance. Therefore, there is a great need to identify novel drugs with more curative effects and fewer side effects to promote sensitivity to TMZ in glioma treatment.

Carnosic acid (CA), a polyphenolic diterpene isolated from Rosemary (Rosmarinus officinalis) or common sage (Salvia officinalis), has various pharmacological effects, including antioxidant [6], anti-inflammatory [7], and anti-cancer properties [8]. For example, in hepatocellular 
carcinoma, CA significantly inhibited cell viability and enhanced apoptosis in vitro [9]. In cervical cancer, CA exerted anti-tumor activity by promoting apoptosis in vitro and in vivo through reactive oxygen species (ROS) production and JNK signaling pathway activation [10]. As in glioma, a previous study showed that $\mathrm{CA}$ at $27.5 \mu \mathrm{M}$ reduced cell survival and induced cell apoptosis via proteasome-mediated degradation of several substrate proteins [11]. In addition to its capacities to directly inhibit tumor progression, CA could synergistically augment the activity of some chemotherapeutic agents in several different types of cancer. CA enhanced trastuzumab inhibition of cell survival and cell migration and induced cell cycle arrest in $\mathrm{ERBB}^{+}{ }^{+}$breast cancer [12]. CA inhibited cell proliferation and enhanced cell apoptosis by increasing intracellular ROS in hepatocellular carcinoma [9]. The CA and fisetin combination treatment led to enhanced inhibition of cell growth by inducing apoptosis in lung cancer [13]. CA enhanced carmustine, lomustine, and $\beta$-lapachone-induced cell growth inhibition and cell cycle arrest in melanoma $[14,15]$. However, the combination effects of CA and TMZ on glioma and the underlying molecular mechanism are still ambiguous.

In this study, we showed that a combination of CA and TMZ synergistically decreased cell viability, cell migration, and colony formation and induced cell cycle arrest by inducing cell apoptosis and autophagy in glioma cancer cells. The cytotoxicity of CA and TMZ co-treatment can be attributed to the downregulation of the PI3K/AKT pathway and the induction of apoptosis and autophagy. Taken together, these data show that the combination of CA and TMZ may provide a new therapeutic strategy for the treatment of glioma.

\section{Materials and methods}

\section{Cell culture and materials}

The glioma cell line U251 was purchased from the Chinese Academy of Science's Cell Bank (Shanghai, China). The glioma cell line LN229 was kindly provided by Prof. Jun Cui at the School of Life Sciences, Sun Yat-sen University, Guangdong, China. The cells were grown in adherent conditions in DMEM supplemented with 10\% FBS, $100 \mathrm{U} / \mathrm{mL}$ penicillin, and $100 \mathrm{mg} / \mathrm{L}$ streptomycin in a $5 \% \mathrm{CO}_{2}$ incubator at $37{ }^{\circ} \mathrm{C}$. CA and TMZ were purchased from Sigma Aldrich (St. Louis, MO, USA).
Fig. 1 Effects of CA, TMZ, and $\mathrm{CA}+\mathrm{TMZ}$ on cell proliferation. a The U251 cell viability was detected by CCK- 8 assay after the cells were treated with the indicated concentrations of CA. b The LN229 cell viability was detected by CCK- 8 assay after the cells were treated with the indicated concentrations of CA. c The $\mathrm{U} 251$ cell viability was detected by CCK- 8 assay after different treatments with TMZ or CA + TMZ. d The LN229 cell viability was detected by CCK- 8 assay after different treatments with TMZ or $\mathrm{CA}+\mathrm{TMZ}$. The results shown are representative of three different experiments. ${ }^{*} \mathrm{p}<0.05$, $* * \mathrm{p}<0.01, * * * \mathrm{p}<0.001$
A
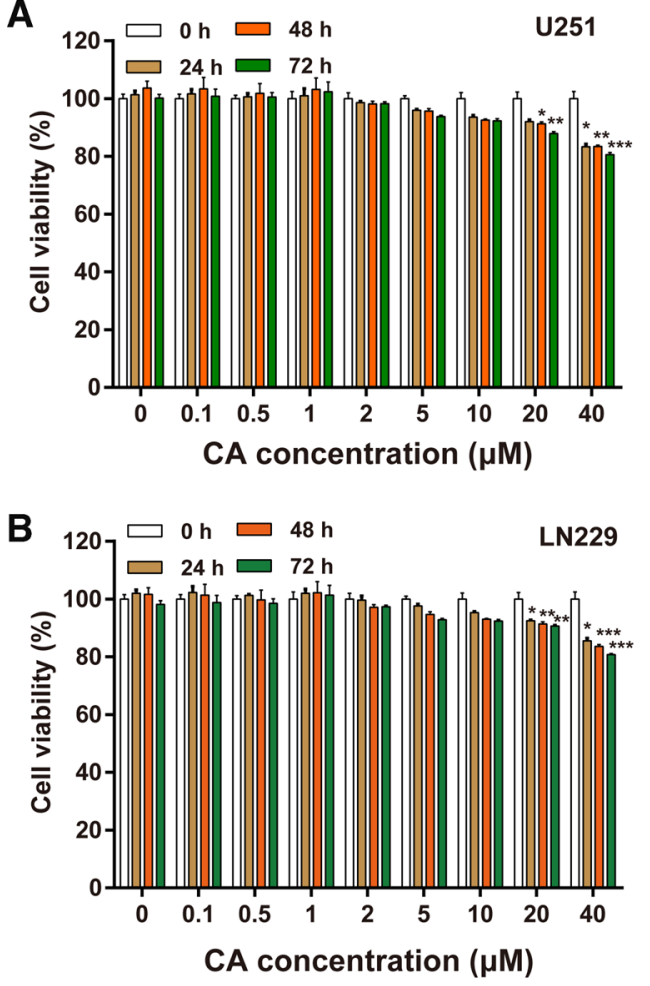

C

U251

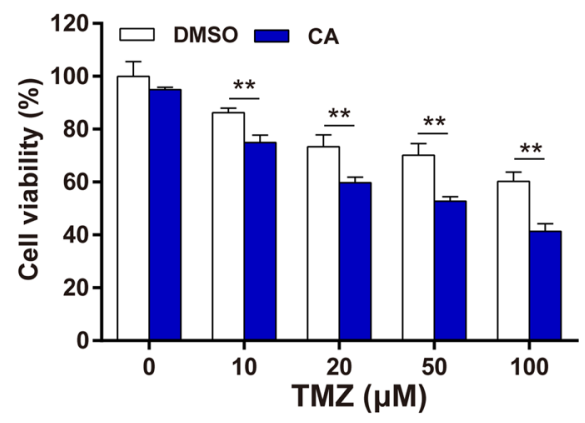

D

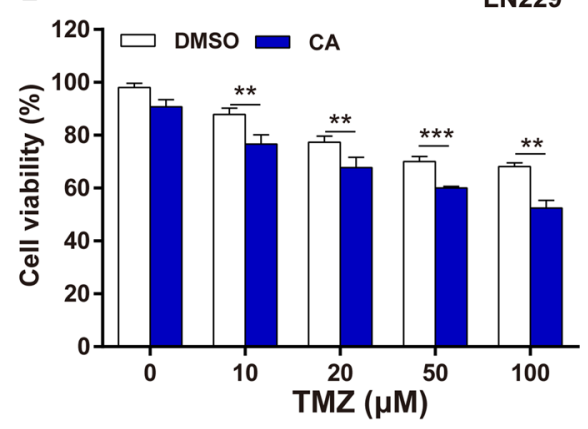


A

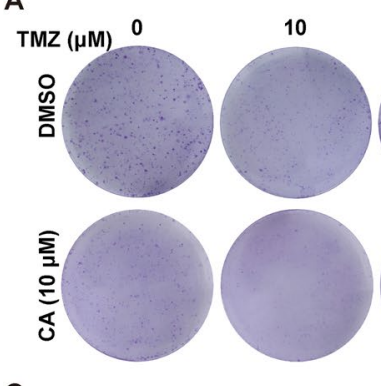

C

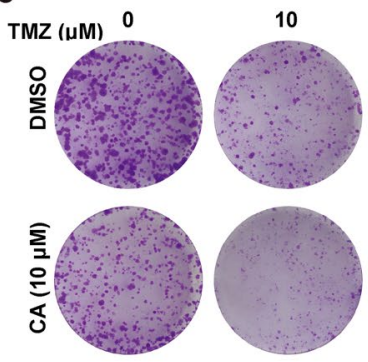

E

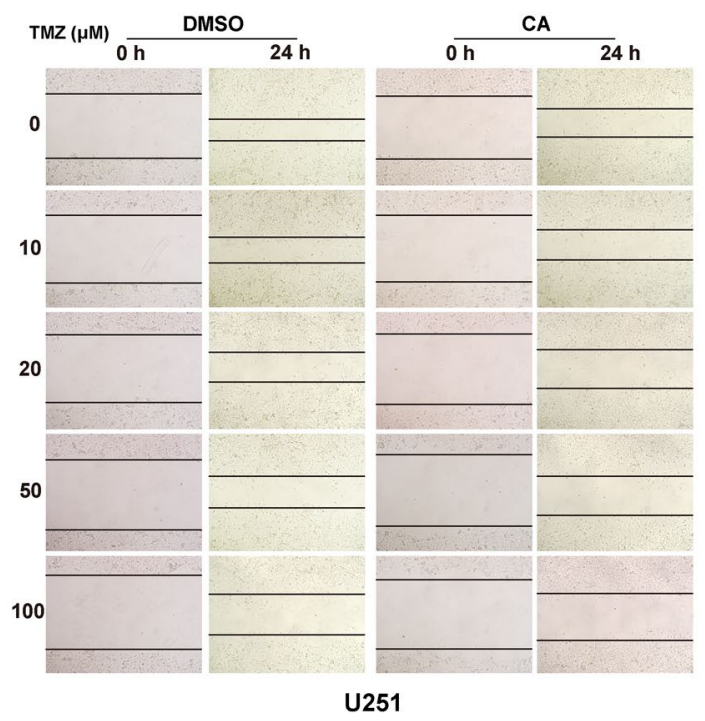

Fig. 2 Effects of TMZ and CA + TMZ on cell colony formation and cell migration. a Representative images of U251 cell colonies after treatment with TMZ or CA + TMZ. b Statistical analysis of colony formation assay results on $\mathrm{U} 251$ cells. $* * \mathrm{p}<0.01, * * * \mathrm{p}<0.001$. c Representative images of LN229 cell colonies after treatment with TMZ or CA + TMZ. d Statistical analysis of colony formation assay results on LN229 cells. **p $<0.01$, ***p $<0.001$. e Representa-
B

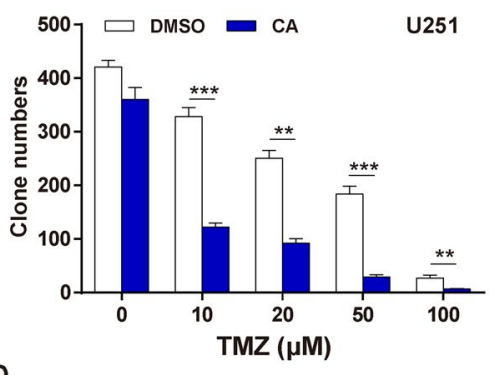

D

100

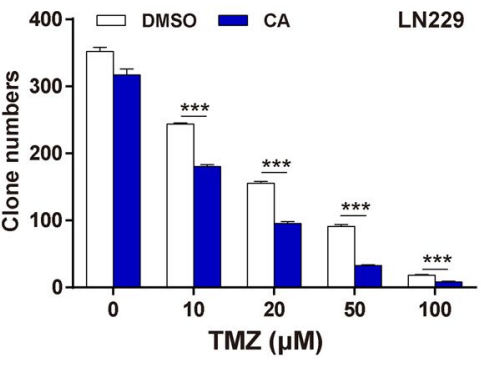

F

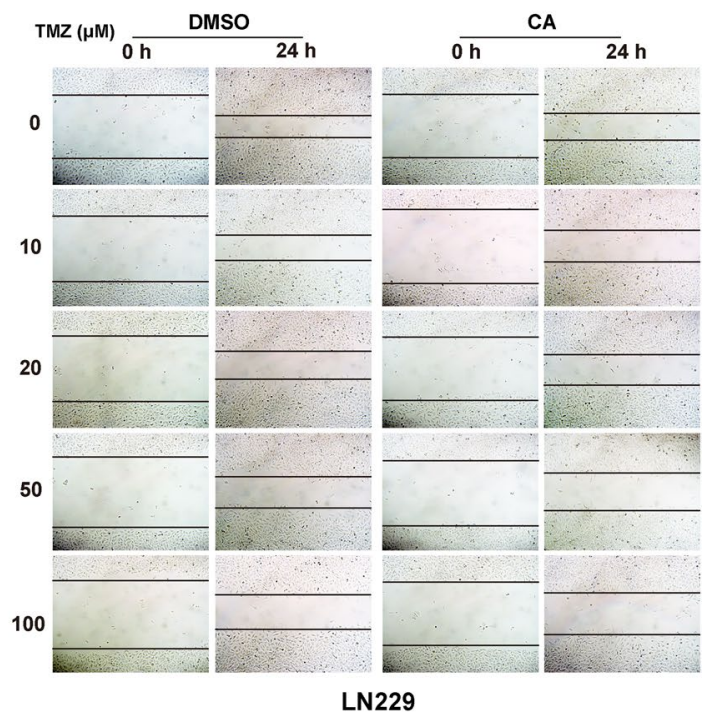

tive images illustrating wound width after treatment with TMZ or $\mathrm{CA}+\mathrm{TMZ}$ on U251 cells. The results shown are representative of three different experiments. f Representative images illustrating wound width after treatment with TMZ or CA + TMZ on LN229 cells. The results shown are representative of three different experiments 


\section{Cell survival assay}

The cells were seeded into a 96-well plate and incubated overnight at $37^{\circ} \mathrm{C}$. The cells were then incubated with CA, $\mathrm{TMZ}$, or CA + TMZ at the indicated concentrations for $24 \mathrm{~h}$, $48 \mathrm{~h}$, and $72 \mathrm{~h}$. Subsequently, each well was filled with $10 \mu \mathrm{L}$ CCK-8 solution (Beyotime, Shanghai, China), and the plate was incubated for $4 \mathrm{~h}$ at $37^{\circ} \mathrm{C}$. The absorbance value was measured at $490 \mathrm{~nm}$ on EL×800 (BioTek, Vermont, USA). The experiment was repeated three times.

\section{Colony formation assay}

The cells were trypsinized and seeded in $60 \mathrm{~mm}$ petri dishes containing 10\% FBS DMEM. The cells were then treated with CA, TMZ, or CA + TMZ at the indicated concentrations and incubated for seven consecutive days. The cells were washed twice with PBS, fixed with $4 \%$ paraformaldehyde and stained by $0.1 \%$ crystal violet. The colonies with over 50 cells were scored under an inverse microscope (IX71, Olympus, Tokyo, Japan). The experiment was repeated at least three times.

\section{Scratch wound-healing assay}

The cells were plated and grown in a 6-well plate until they reached $>80 \%$ confluence. A scratching wound was formed using a $200 \mu \mathrm{L}$ sterile pipette tip in the middle of the cell monolayer. Cells were then washed with PBS to remove the debris and were cultured with complete medium containing CA, TMZ, or both at the indicated concentrations. The representative images were taken at $0 \mathrm{~h}$ and $24 \mathrm{~h}$ under an inverse microscope (IX71, Olympus, Tokyo, Japan).

\section{Cell cycle and apoptosis assays}

The cells were seeded into 6-well plates and grown until $>80 \%$ confluence. The cells were then treated with CA, $\mathrm{TMZ}$, or CA + TMZ at the indicated concentrations for $24 \mathrm{~h}$. For cell cycle analysis, cells were trypsinized and fixed in $70 \%$ ethanol overnight. The cells were stained according to the manufacturer's protocol using the cell cycle analysis kit (Beyotime, Shanghai, China) and detected by flow cytometry (Guava EasyCyte 6HT-2L, Merck Millipore, Darmstadt, Germany). For cell apoptosis analysis, the cells were stained with the cell apoptosis analysis kit (Beyotime, Shanghai, China) according to the manufacturer's protocol and detected by flow cytometry (Guava EasyCyte 6HT-2L, Merck Millipore, Darmstadt, Germany).

\section{Immunofluorescence analysis}

The cells were seeded on cover slips in a 6-well plate and then were treated with CA, TMZ, or both at the indicated concentrations for $24 \mathrm{~h}$. The cells were fixed with $4 \%$ paraformaldehyde for 40 min under room temperature and were then stained with LC3 antibody (1:100, \#2775, Cell Signalling Technology, MA, USA). The nuclei were counterstained with $0.1 \mu \mathrm{g} / \mathrm{ml}$ DAPI. Cells were then stained with a secondary antibody (1:1000, \#A-11034, Thermo Fisher Scientific, MA, USA). The coverslips were viewed using confocal microscopy (FV10i, Olympus, Tokyo, Japan) with appropriate filters.

\section{Western blot analysis}

The cells were cultured in a 6-well plate and were treated with CA, TMZ, or both at the indicated concentrations for $24 \mathrm{~h}$. The cells were lysed in RIPA buffer (Beyotime, Shanghai, China) containing 1\% protease and phosphatase inhibitor cocktail (Roche, Basel, Switzerland). Supernatant was collected after centrifuging at 12,000 rpm for $15 \mathrm{~min}$ at $4{ }^{\circ} \mathrm{C}$. The protein concentration was determined using a Standard BCA Protein Assay Kit (Thermo Fisher Scientific, MA, USA). Western blot analysis was performed according to the standard protocol [16]. The primary antibodies against LC3 (\#2775), Cyclin B1 (\#4138), PARP (\#9532), Caspase-3 (\#9665), cleaved Caspase-3 (\#9664), AKT (\#4691), p-AKT (\#4060), p62 (\#8025), and $\beta$-actin (\#3700) were purchased from Cell Signaling Technology (MA, USA). Chemiluminescent signals were detected using an ECL plus kit (Thermo Fisher Scientific, MA, USA) on a ChemiDoc Touch Imaging System (BioRad, CA, USA).

\section{Statistical analysis}

Data were expressed as the mean \pm SD and were analyzed by GraphPad Prism 5.0 software. One-way analysis of variance (ANOVA) and two-tailed Student's t-test were employed to compare different groups. $\mathrm{p}<0.05$ was considered statistically significant. 

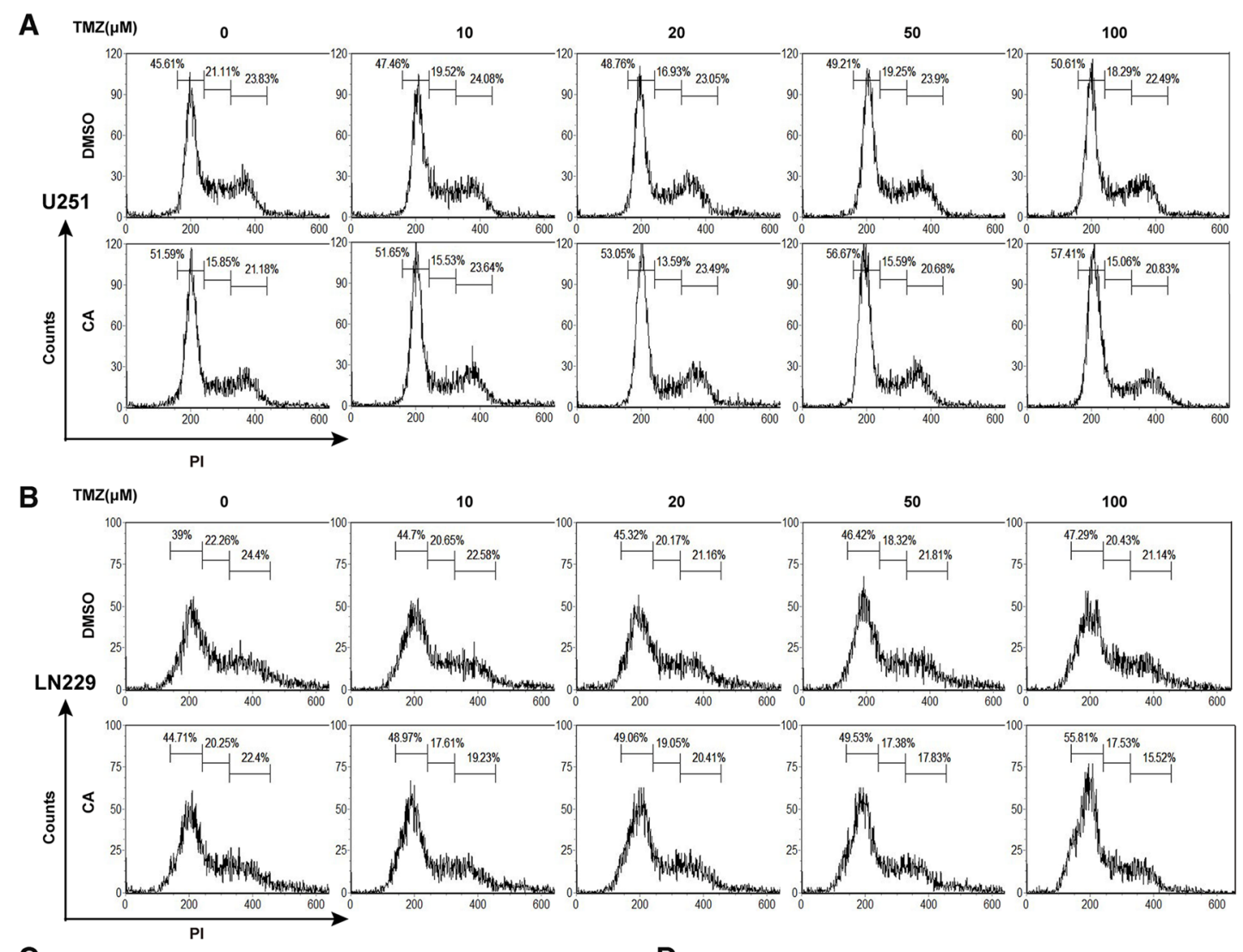

C

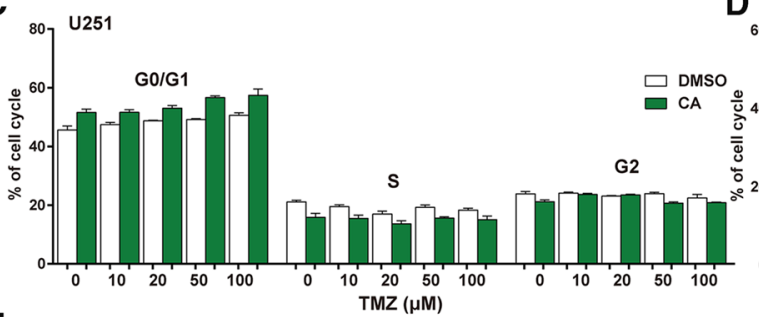

$D_{60}{ }^{\text {LN229 }}$

E

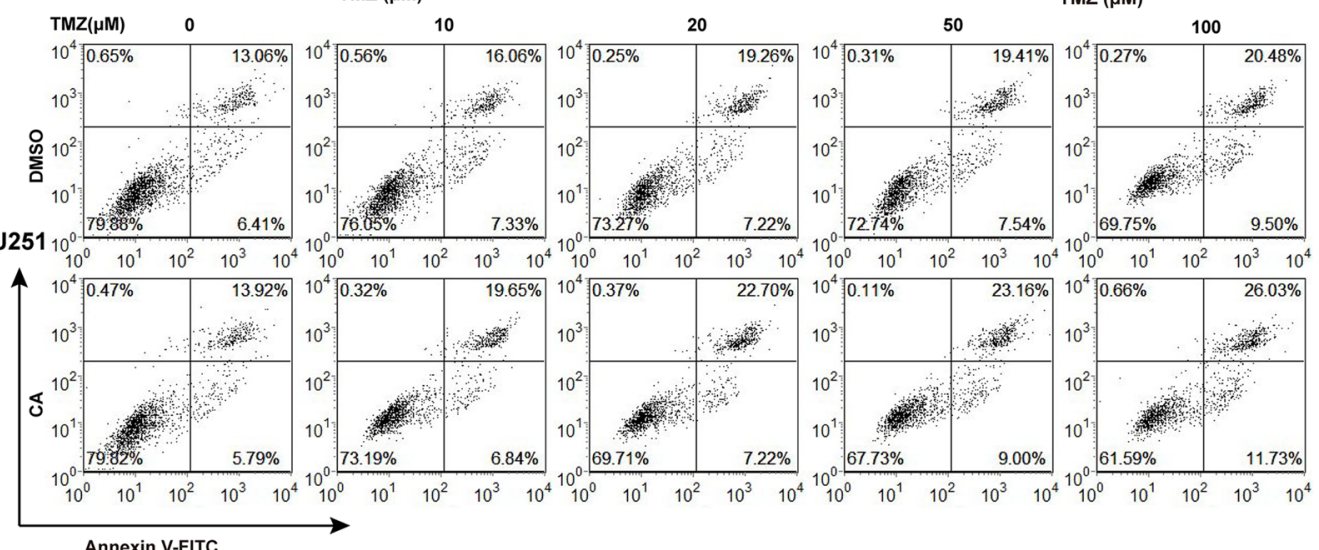

Fig. 3 Effects of TMZ and CA + TMZ on glioma cell cycle and cell apoptosis. The cells were treated with TMZ or CA + TMZ at indicated concentrations for $24 \mathrm{~h}$. The experiments were repeated for three times. a The cell cycle stage of U251 cells was evaluated by flow cytometry. b The cell cycle stage of LN229 cells was evaluated by flow cytometry. c Statistical analysis of the cell cycle assay results on U251 cells. d Statistical analysis of the cell cycle assay results on LN229 cells. e The cell apoptosis was evaluated by flow cytometry on
$\mathrm{U} 251$ cells. The apoptosis rate $=\left(\right.$ Annexin $\mathrm{V}^{+} \mathrm{PI}^{+}+$Annexin $\left.\mathrm{V}^{+} \mathrm{PI}^{-}\right) /$ total cells $\times 100 \%$. f The cell apoptosis was evaluated by flow cytometry on LN229 cells. The apoptosis rate $=\left(\right.$ Annexin $\mathrm{V}^{+} \mathrm{PI}^{+}+$Annexin $\mathrm{V}^{+} \mathrm{PI}^{-}$)/total cells $\times 100 \%$. g Statistical analysis of cell apoptosis assay results from U251 cells. $* * \mathrm{p}<0.01$. h Statistical analysis of cell apoptosis assay results from LN229 cells. ${ }^{*} \mathrm{p}<0.05, * * \mathrm{p}<0.01$, $* * * \mathrm{p}<0.001$ 


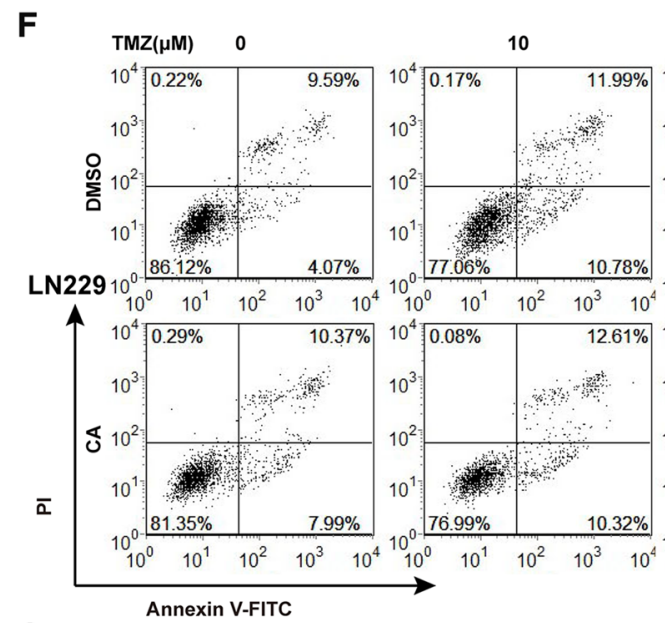

G

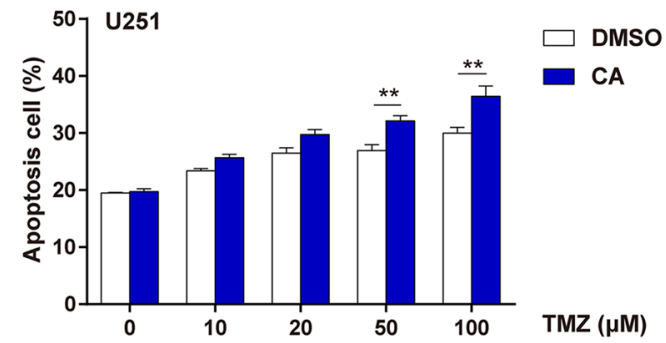

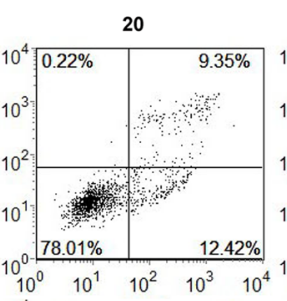
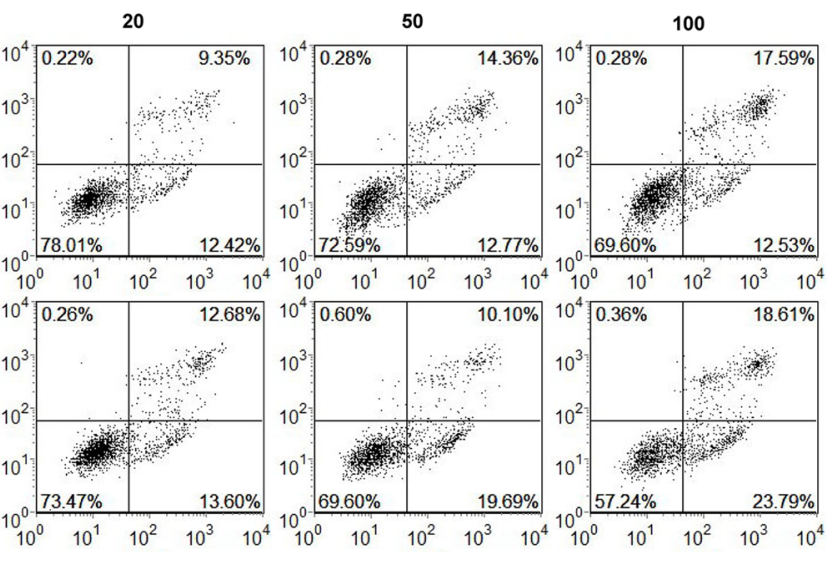

H

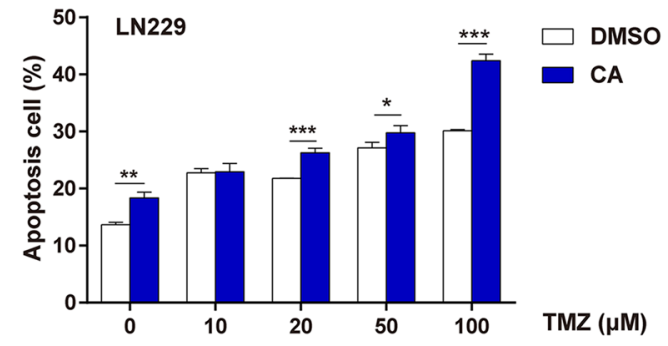

Fig. 3 (continued)

\section{Results}

\section{CA and TMZ combination inhibited cell viability}

To determine the antitumor effects of CA and TMZ combination in glioma, the U251 and LN229 glioma cancer cells were treated with CA, TMZ or TMZ + CA at indicated concentrations. As shown in Fig. 1a, b, no obvious cytotoxicity was observed in U251 cells as well as in LN229 cells after CA administration at the concentration of $10 \mu \mathrm{M}$ or lower. Thus a CA concentration of $10 \mu \mathrm{M}$ was selected in the subsequent experiments. As expected, treatment with TMZ $(0-100 \mu \mathrm{M})$ significantly reduced cell viability in a dosedependent manner in both U251 and LN229 cells (Fig. 1c, d). Moreover, after the combination treatment of CA with TMZ, the inhibitory effect of TMZ on U251 and LN229 cells was significantly increased (Fig. 1c, d). These results indicated that CA combined with TMZ has an enhanced antitumor effect in glioma.

\section{CA and TMZ combination inhibited cell colony formation and cell migration}

A colony formation assay was performed to evaluate cell division capabilities under different treatments. As shown in Fig. 2a, TMZ alone significantly inhibited cell clone formation in a dose-dependent manner, while co-treatment with CA prominently enhanced the inhibitory effect of TMZ on clone formation. The statistical analysis is shown in Fig. 2b. The inhibitory effects of CA and TMZ on cell clone formation were similar on LN229 cells (Fig. 2c) and the statistical analysis was performed in Fig. 2d. Next, a woundhealing assay was conducted to investigate the migration capability of cells following treatment. As shown in Fig. 2e, the speed of wound closure was significantly hindered by TMZ with increased concentration. The CA and TMZ combination showed enhanced effects in controlling the wound width of U251 cells. Similar results were observed in LN229 cells as shown in Fig. 2f. These results indicated that CA combined with TMZ has an inhibitory effect on glioma colony formation and cell migration.

\section{CA and TMZ combination arrested cell cycle progression and promoted cell apoptosis}

To explore the potential underlying mechanism by which CA could potentiate TMZ-induced cell death in glioma, cell cycle and cell apoptosis assays were performed and assessed by flow cytometry on U251 and LN229 cells. The cell cycle assay results showed that TMZ could induce a moderate G0/G1 phase accumulation and a slight $S$ and $\mathrm{G} 2 / \mathrm{M}$ phase reduction even at the highest concentration 
in our experiment, while the combination treatment could potentiate the effects induced by TMZ alone in both U251 and LN229 cells (Fig. 3a, b). The statistical analysis for the cell cycle assay on U251 cells is shown in Fig. 3c and that for LN229 cells is shown in Fig. 3d. The cell apoptosis assay showed that the rate of cellular apoptosis induced by TMZ increased in a concentration-dependent manner. The combination treatment had a higher apoptosis rate in both U251 and LN229 cells when compared with TMZ alone. In U251 cells, the apoptosis rate induced by $100 \mu \mathrm{M}$ TMZ was $29.98 \%$ compared to an apoptosis rate of $37.76 \%$ when treated with TMZ and CA in combination (Fig. 3e). In LN229 cells, the apoptosis rate induced by $100 \mu \mathrm{M}$ TMZ was $30.12 \%$ compared to an apoptosis rate of $42.40 \%$ when treated with TMZ and CA in combination (Fig. 3f). The statistical analysis for the U251 apoptosis assay is shown in Fig. $3 \mathrm{~g}$ and that for the LN229 is shown in Fig. $3 \mathrm{~h}$.

To explore the potential molecular mechanism of CAenhanced cell cycle arrest and cell apoptosis, western blot analysis was carried out. As shown in Fig. 4a, in U251 cells Cyclin B1 was slightly downregulated, while cleaved PARP and cleaved Caspase-3 were moderately increased when treated with TMZ alone. Furthermore, CA could potentiate these changes when CA and TMZ combination treatment was used. The statistical analysis for Cyclin B1, PARP, and Caspase- 3 are shown in Fig. 4b-d, respectively. Similar results were observed in LN229 cells as shown in Fig. 4e-h. Taken together, these results indicated that CA and TMZ combination could arrest cell cycle and promote cell apoptosis in glioma.

\section{CA and TMZ combination induced autophagy}

To determine the effect of CA and TMZ combination treatment on autophagy activation in glioma cancer cells, immunofluorescence was performed to detect LC3-II, an important component of the autophagosome. As shown in Fig. 5a, the combination treatment induced more coarse dots and punctate staining than with treatment of TMZ alone. The statistical analysis for this assay is shown in Fig. 5b. Similar results were observed in LN229 cells as shown in Fig. 5c, d. To reveal the molecular mechanism of this autophagy activation, some key autophagy-associated proteins were examined by western blot analysis. Obvious LC3-II accumulation and p62 reduction were observed in TMZ-treated U251 cells. Moreover, these phenomena were more apparent following CA addition (Fig. 5e). As the PI3K/AKT pathway is a key regulator in autophagy, the protein expression levels of p-AKT and AKT were detected. As shown in Fig. 5e, combination treatment significantly decreased the expression of p-AKT compared with TMZ alone in U251 cells. The statistical analyses for the levels of p-AKT/AKT, p62, and LC3-II/LC3-I are shown in Fig. 5f-h, respectively.
Similar results were observed in LN229 cells as shown in Fig. 5i-1. There also existed some differences between U251 and LN229 cells when they were treated with CA, TMZ or both. For example, the ratio of the p-AKT/AKT in LN229 cells was not downregulated until the TMZ concentration greater than $20 \mu \mathrm{M}$. All the above results suggested that the combination of CA and TMZ could induce autophagy in glioma cancer cells.

\section{Discussion}

The standard chemotherapy for glioma treatment with TMZ has some limitations, such as severe side effects and poor drug response $[17,18]$. The need for novel drugs that can enhance TMZ bioavailability and overcome chemoresistance is urgent in clinical application. In this study, we demonstrated that the natural, polyphenolic drug CA may enhance the anticancer effect of TMZ by promoting the apoptosis and autophagy induced by TMZ.

CA has been widely proposed as a potential therapeutic compound in cancer treatment and prevention due to its ability to modulate cell growth and differentiation via different key molecules and signaling pathways [19]. CA impedes cell growth, induces cell cycle arrest, promotes apoptosis, and decreases mitochondrial membrane potential; CA has been shown to induce autophagy in various cancers: by enhancing p21 expression in melanoma [14] by inhibiting the AKT/ mTOR pathway in hepatocellular carcinoma [20], by activating the JNK pathway in cervical cancer [10], by suppressing the Src/STAT3 pathway in renal carcinoma [21], by inhibiting the STAT3 pathway in colon cancer [22], by downregulating miR-15b in pancreatic cancer [23], and by modulating the $\mathrm{AKT} / \mathrm{IKK} / \mathrm{NF}-\kappa \mathrm{B}$ pathway in prostate carcinoma [24]. In glioma, Cortese et al. found that $\mathrm{CA}$ induced cell growth arrest and apoptosis via Cyclin B1, RB and SOX2 downregulation in glioblastoma [11]. CA may also function by enhancing the antitumor effects of current chemotherapeutic agents [19]. CA enhances the cellular cytotoxicity induced by $\beta$-lapachone in melanoma [15], by tamoxifen [25] and doxorubicin [26] in breast cancer, and by adriamycin [27] and arsenic trioxide [28] in leukemia. In this study, though TMZ could inhibit glioma cell growth, TMZ in combination with low-dose CA was more effective than TMZ alone. It is worth noting that $\mathrm{CA}$ at the concentration used in this work had little cytotoxic effect when used alone.

Apoptosis and autophagy are two distinct self-destructive processes that determine cell fate under physiological as well as pathological conditions [29]. Apoptosis is a process of programmed cell death that is executed by activated caspases and specific enzymes whose activation or inactivation lead to characteristic cell changes and cell death [30]. The apoptotic cascade is divided into 
A

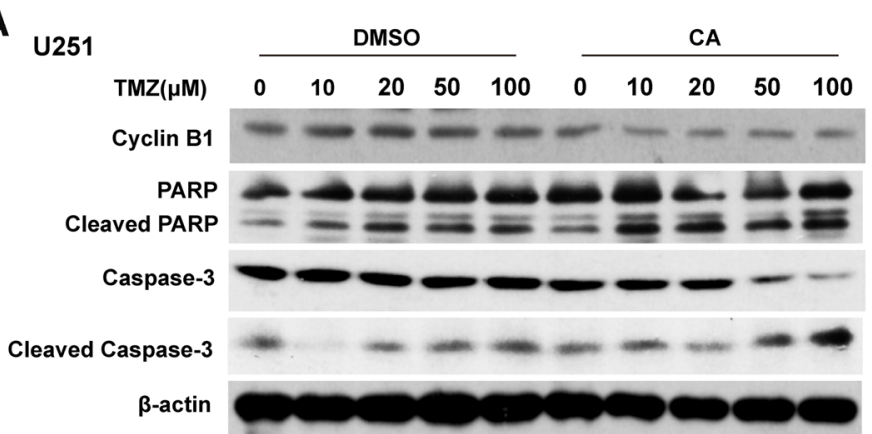

C

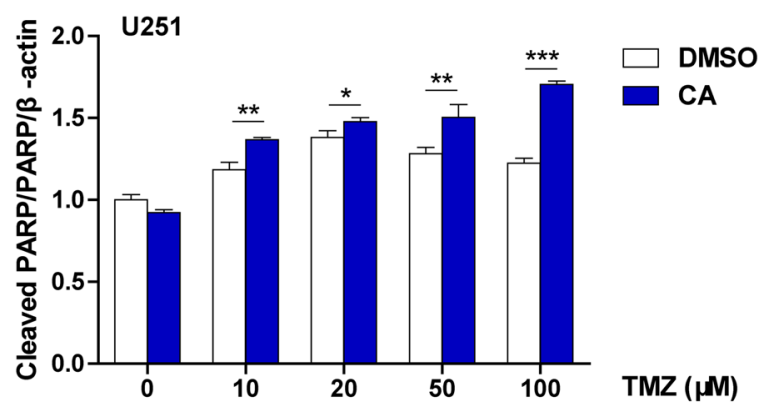

E

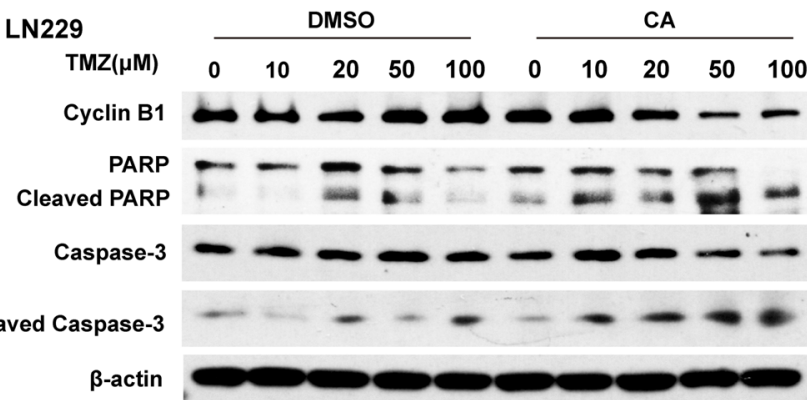

G

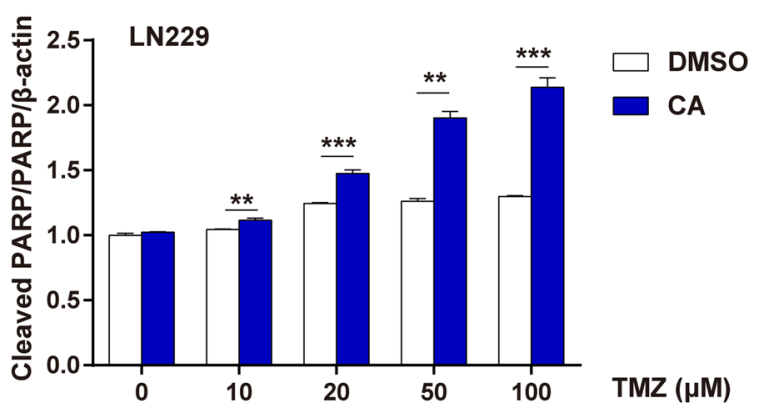

B

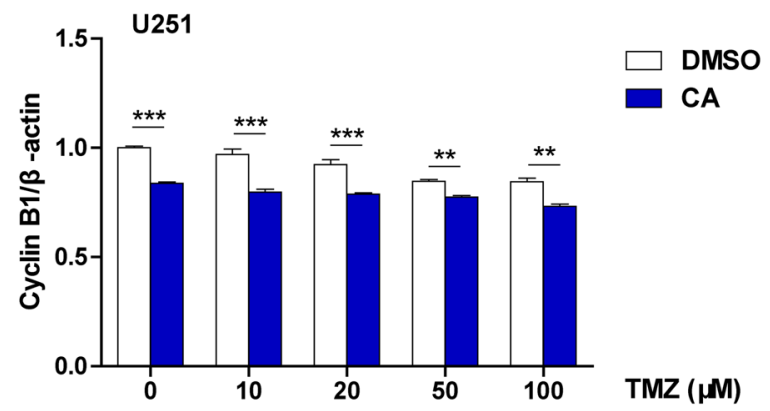

D.

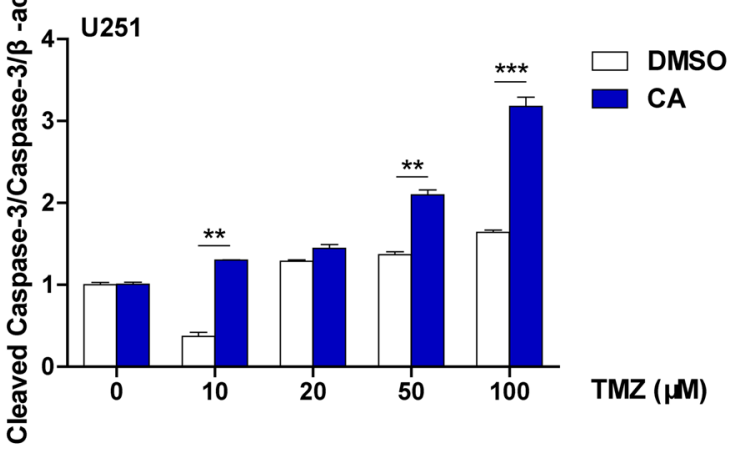

$\mathbf{F}$
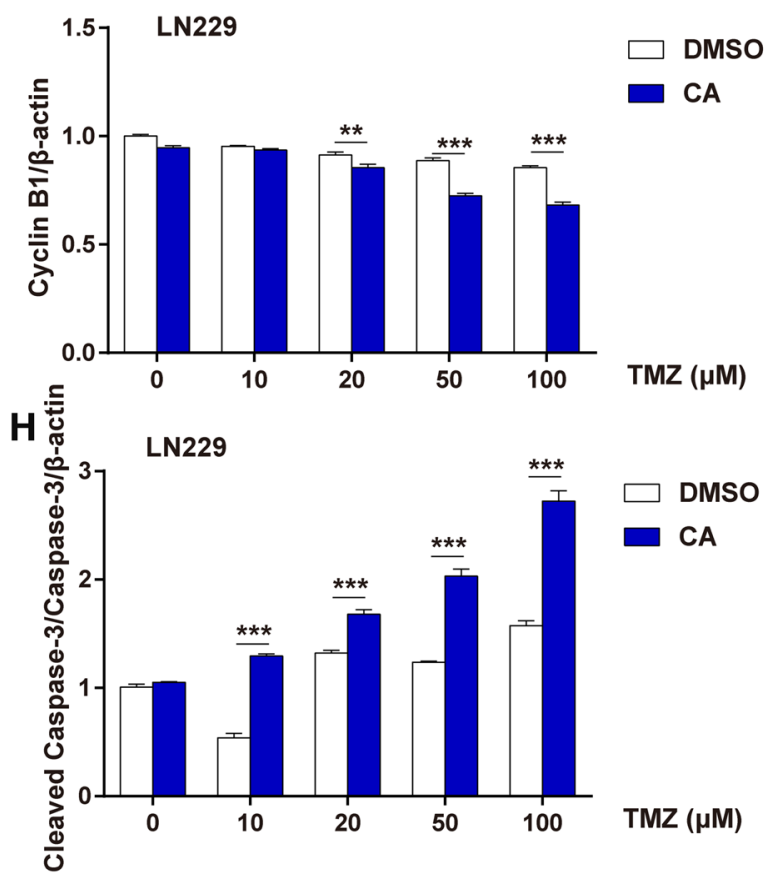
4Fig. 4 Effects of TMZ and CA+TMZ on glioma cell cycle and cell apoptosis related proteins. The cells were treated with TMZ or $\mathrm{CA}+\mathrm{TMZ}$ at indicated concentrations for 24. a The protein levels of Cyclin B1, PARP, cleaved PARP, Caspase-3, and cleaved Caspase-3 in U251 cells were evaluated by western blot analysis. b Statistical analysis of Cyclin B1 protein level in U251 cells from western blot results. Relative Cyclin B1 protein level was determined by signal quantification and was normalized to $\beta$-actin. The relative Cyclin B1 protein level in the DMSO treatment group without TMZ or CA was arbitrarily set as 1 . c Statistical analysis of cleaved PARP/PARP in U251 cells from western blot results. Relative cleaved PARP and PARP protein levels were determined by signal quantification and were normalized to $\beta$-actin. The ratio of cleaved PARP/PARP in the DMSO treatment group without TMZ or CA was arbitrarily set as 1 . d Statistical analysis of the cleaved Caspase-3/Caspase-3 protein ratio in U251 cells from western blot results. Relative cleaved Caspase-3 and Caspase- 3 protein levels were determined by signal quantification and were normalized to $\beta$-actin. The ratio of cleaved Caspase-3/ Caspase- 3 in the DMSO treatment group without TMZ or CA was arbitrarily set as 1 . e The protein levels of Cyclin B1, PARP, cleaved PARP, Caspase-3, and cleaved Caspase- 3 in LN229 cells were evaluated by western blot analysis. f Statistical analysis of Cyclin B1 protein level in LN229 cells from western blot results. Relative Cyclin B1 protein level was determined by signal quantification and was normalized to $\beta$-actin. The relative Cyclin B1 protein level in the DMSO treatment group without TMZ or CA was arbitrarily set as 1. g Statistical analysis of cleaved PARP/PARP in LN229 cells from western blot results. Relative cleaved PARP and PARP protein levels were determined by signal quantification and were normalized to $\beta$-actin. The ratio of cleaved PARP/PARP in the DMSO treatment group without TMZ or CA was arbitrarily set as 1 . h Statistical analysis of the cleaved Caspase-3/Caspase-3 protein ratio in LN229 cells from western blot results. Relative cleaved Caspase- 3 and Caspase- 3 protein levels were determined by signal quantification and were normalized to $\beta$-actin. The ratio of cleaved Caspase-3/Caspase- 3 in the DMSO treatment group without TMZ or CA was arbitrarily set as 1 . The results shown are representative of three different experiments

the extrinsic apoptotic pathway and the intrinsic apoptotic pathway [31]. The extrinsic pathway is activated by death receptors and executed by initiator caspases and effector caspases (Caspase-3, Caspase-6, and Caspase-7) [32]. During drug-induced apoptosis, PARP is cleaved into fragments, and thus, cleaved PARP is usually used as a marker of apoptosis [33]. Our results showed that
TMZ can increase the protein level of cleaved PARP and cleaved Caspase-3, while CA can enhance the effects of TMZ on PARP and Caspase- 3 in glioma, indicating the potential of CA to induce apoptosis in glioma. However, it was worthy to note that the ratio of the cleaved Caspase-3/ Caspase- 3 in the $10 \mu \mathrm{M}$ TMZ group was consistently lower than the other groups in our experiments. The underlying mechanism for this phenomenon still needs to be explored. Autophagy is the natural and orderly process to eradicate cytoplasmic organelles or unused proteins and thus to maintain cellular homeostasis [34]. As an important receptor in autophagy, LC3-II is considered the molecular marker for the autophagosome [35]. p62, a selective autophagy adaptor/receptor, binds with ubiquitinated proteins and LC3 for engulfment [36]. In our study, the combination treatment markedly increased the protein level of LC3-II and reduced the protein level of p62, suggesting that the autophagy induction was enhanced. Furthermore, the phosphorylation of AKT was decreased following CA treatment, suggesting that CA inhibits the PI3K/AKT signaling pathway, which is an important negative regulator of autophagy [37]. It is worth noting that there is a complex interaction between autophagy and apoptosis, especially in cancer etiology and treatment [38]. In our study, the CA and TMZ combination could induce apoptosis and autophagy in glioma cancer cells. However, the underlying mechanism of the connection between autophagy and apoptosis under the combination treatment remains unresolved. Our study may also have some limitations; for example, the signaling pathways influenced by CA were not exhaustively investigated. These issues can be resolved in our future studies.

In conclusion, the present study demonstrates for the first time that CA increases the sensitivity of glioma cancer cells to TMZ via the induction of apoptosis and autophagy. A combination therapy of TMZ with CA may be a promising therapeutic strategy for glioma in the future. 

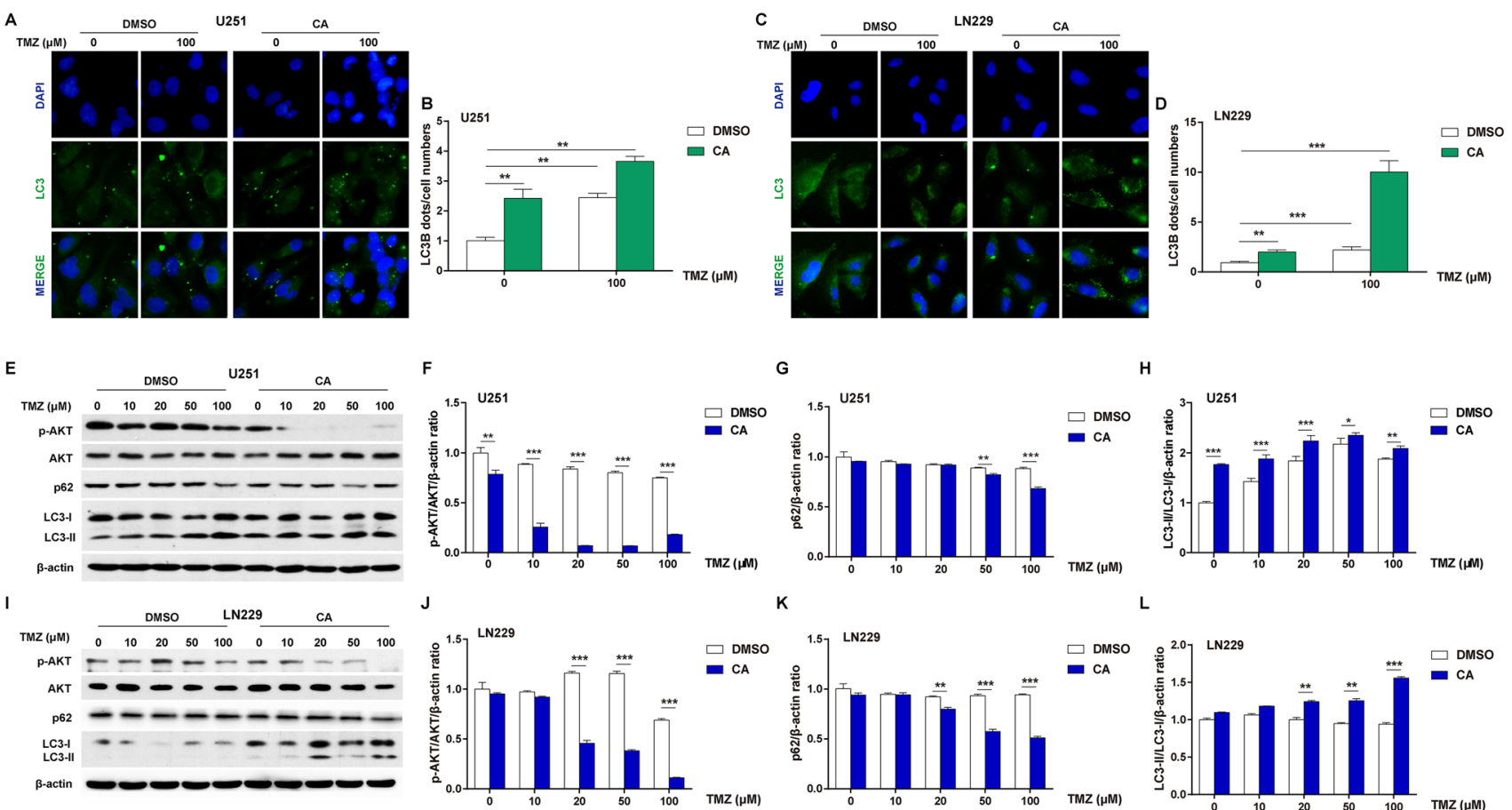

$\mathrm{L}$
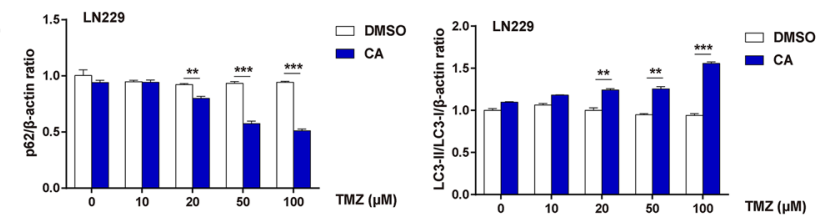

Fig. 5 Effects of TMZ and CA + TMZ on glioma cell autophagy. The cells were treated with TMZ or CA + TMZ at indicated concentrations for $24 \mathrm{~h}$. a The immunofluorescence analysis of LC3 in U251 cells. Nuclei were stained by DAPI. b The statistical analysis of number of LC3 dots per cell in U251 cells. $\mathbf{c}$ The immunofluorescence analysis of LC3 in LN229 cells. Nuclei were stained by DAPI. d The statistical analysis of number of LC3 dots per cell in LN229 cells. e The protein levels of p-AKT, AKT, p62, and LC3 in U251 cells were evaluated by western blot analysis. f Statistical analysis of p-AKT/AKT ratio in U251 cells from western blot results. Relative p-AKT and AKT protein levels were determined by signal quantification and were normalized to $\beta$-actin. The ratio of $\mathrm{p}$-AKT/AKT in the DMSO treatment group without TMZ or CA was arbitrarily set as 1. g Statistical analysis of p62 in U251 cells from western blot results. Relative p62 protein level was determined by signal quantification and was normalized to $\beta$-actin. The relative p62 protein level in the DMSO treatment group without TMZ or CA was arbitrarily set as 1 . $\mathbf{h}$ Statistical analysis of LC3-II/LC3-I in U251 cells from west-

Funding This study was funded by the National Natural Science Foundation of China (81302197), the Changzhou Science and Technology Support Program (CE20165048), the Changzhou High-Level Medical Talents Training Project (2016CZBJ006), and the Changzhou Municipal Commissions of Health and Family Planning Major Scientific and Technological Project (ZD201620).

\section{Compliance with ethical standards}

Conflict of interest The authors declare that they have no conflict of interest.

Ethical approval All procedures performed in studies involving human participants were in accordance with the ethical standards of the institutional and/or national research committee and with the 1964 Helsinki declaration and its later amendments or comparable ethical standards. ern blot results. Relative LC3-II and LC3-I protein levels were determined by signal quantification and were normalized to $\beta$-actin. The ratio of LC3-II/LC3-I in the DMSO treatment group without TMZ or CA was arbitrarily set as 1 . i The protein levels of p-AKT, AKT, p62, and LC3 in LN229 cells were evaluated by western blot analysis. $\mathbf{j}$ Statistical analysis of p-AKT/AKT ratio in LN229 cells from western blot results. Relative p-AKT and AKT protein levels were determined by signal quantification and were normalized to $\beta$-actin. The ratio of p-AKT/AKT in the DMSO treatment group without TMZ or CA was arbitrarily set as 1 . k Statistical analysis of p62 in LN229 cells from western blot results. Relative p62 protein level was determined by signal quantification and was normalized to $\beta$-actin. The relative p62 protein level in the DMSO treatment group without TMZ or CA was arbitrarily set as 1 . I Statistical analysis of LC3-II/LC3-I in LN229 cells from western blot results. Relative LC3-II and LC3-I protein levels were determined by signal quantification and were normalized to $\beta$-actin. The ratio of LC3-II/LC3-I in the DMSO treatment group without TMZ or CA was arbitrarily set as 1

Informed consent Informed consent was obtained from all individual participants included in the study.

OpenAccess This article is distributed under the terms of the Creative Commons Attribution 4.0 International License (http://creativeco mmons.org/licenses/by/4.0/), which permits unrestricted use, distribution, and reproduction in any medium, provided you give appropriate credit to the original author(s) and the source, provide a link to the Creative Commons license, and indicate if changes were made.

\section{References}

1. Ostrom QT, Gittleman H, Liao P, Vecchione-Koval T, Wolinsky Y, Kruchko C, Barnholtz-Sloan JS (2017) CBTRUS statistical 
report: primary brain and other central nervous system tumors diagnosed in the United States in 2010-2014. Neuro Oncol 19(suppl_5):v1-v88. https://doi.org/10.1093/neuonc/nox158

2. Gladson CL, Prayson RA, Liu WM (2010) The pathobiology of glioma tumors. Annu Rev Pathol 5:33-50. https://doi.org/10. 1146/annurev-pathol-121808-102109

3. Stupp R, Mason WP, van den Bent MJ, Weller M, Fisher B, Taphoorn MJ, Belanger K, Brandes AA, Marosi C, Bogdahn U, Curschmann J, Janzer RC, Ludwin SK, Gorlia T, Allgeier A, Lacombe D, Cairncross JG, Eisenhauer E, Mirimanoff RO, European Organisation for R, Treatment of Cancer Brain T, Radiotherapy G, National Cancer Institute of Canada Clinical Trials G (2005) Radiotherapy plus concomitant and adjuvant temozolomide for glioblastoma. N Engl J Med 352(10):987996. https://doi.org/10.1056/NEJMoa043330

4. Fukushima T, Takeshima H, Kataoka H (2009) Anti-glioma therapy with temozolomide and status of the DNA-repair gene MGMT. Anticancer Res 29(11):4845-4854

5. Agarwala SS, Kirkwood JM (2000) Temozolomide, a novel alkylating agent with activity in the central nervous system, may improve the treatment of advanced metastatic melanoma. Oncologist 5(2):144-151

6. Sahu BD, Putcha UK, Kuncha M, Rachamalla SS, Sistla R (2014) Carnosic acid promotes myocardial antioxidant response and prevents isoproterenol-induced myocardial oxidative stress and apoptosis in mice. Mol Cell Biochem 394(1-2):163-176. https://doi.org/10.1007/s11010-014-2092-5

7. Yang N, Xia Z, Shao N, Li B, Xue L, Peng Y, Zhi F, Yang Y (2017) Carnosic acid prevents dextran sulfate sodium-induced acute colitis associated with the regulation of the Keap1/ Nrf2 pathway. Sci Rep 7(1):11036. https://doi.org/10.1038/ s41598-017-11408-5

8. Moore J, Yousef M, Tsiani E (2016) Anticancer effects of rosemary (Rosmarinus officinalis L.) extract and rosemary extract polyphenols. Nutrients. https://doi.org/10.3390/nu8110731

9. Zhang X, Chen Y, Cai G, Li X, Wang D (2017) Carnosic acid induces apoptosis of hepatocellular carcinoma cells via ROSmediated mitochondrial pathway. Chem Biol Interact 277:91100. https://doi.org/10.1016/j.cbi.2017.09.005

10. Su K, Wang CF, Zhang Y, Cai YJ, Zhang YY, Zhao Q (2016) The inhibitory effects of carnosic acid on cervical cancer cells growth by promoting apoptosis via ROS-regulated signaling pathway. Biomed Pharmacother 82:180-191. https://doi.org/10. 1016/j.biopha.2016.04.056

11. Cortese K, Daga A, Monticone M, Tavella S, Stefanelli A, Aiello C, Bisio A, Bellese G, Castagnola P (2016) Carnosic acid induces proteasomal degradation of cyclin B1, RB and SOX2 along with cell growth arrest and apoptosis in GBM cells. Phytomedicine 23(7):679-685. https://doi.org/10.1016/j.phymed. 2016.03.007

12. D'Alesio C, Bellese G, Gagliani MC, Aiello C, Grasselli E, Marcocci G, Bisio A, Tavella S, Daniele T, Cortese K, Castagnola $P$ (2017) Cooperative antitumor activities of carnosic acid and Trastuzumab in ERBB2(+) breast cancer cells. J Exp Clin Cancer Res 36(1):154. https://doi.org/10.1186/s13046-017-0615-0

13. Shi B, Wang LF, Meng WS, Chen L, Meng ZL (2017) Carnosic acid and fisetin combination therapy enhances inhibition of lung cancer through apoptosis induction. Int J Oncol 50(6):21232135. https://doi.org/10.3892/ijo.2017.3970

14. Lin KI, Lin CC, Kuo SM, Lai JC, Wang YQ, You HL, Hsu ML, Chen CH, Shiu LY (2018) Carnosic acid impedes cell growth and enhances anti-cancer effects of carmustine and lomustine in melanoma. Biosci Rep. https://doi.org/10.1042/BSR20180005

15. Arakawa N, Okubo A, Yasuhira S, Takahashi K, Amano H, Akasaka T, Masuda T, Shibazaki M, Maesawa C (2018) Carnosic acid, an inducer of $\mathrm{NAD}(\mathrm{P}) \mathrm{H}$ quinone oxidoreductase 1 , enhances the cytotoxicity of beta-lapachone in melanoma cell lines. Oncol Lett 15(2):2393-2400. https://doi.org/10.3892/ol. 2017.7618

16. Deng D, Wang L, Chen Y, Li B, Xue L, Shao N, Wang Q, Xia X, Yang Y, Zhi F (2016) MicroRNA-124-3p regulates cell proliferation, invasion, apoptosis, and bioenergetics by targeting PIM1 in astrocytoma. Cancer Sci 107(7):899-907. https://doi. org/10.1111/cas.12946

17. Vaios EJ, Nahed BV, Muzikansky A, Fathi AT, Dietrich J (2017) Bone marrow response as a potential biomarker of outcomes in glioblastoma patients. J Neurosurg 127(1):132-138. https://doi. org/10.3171/2016.7.JNS16609

18. Osuka S, Van Meir EG (2017) Overcoming therapeutic resistance in glioblastoma: the way forward. J Clin Invest 127(2):415-426. https://doi.org/10.1172/JCI89587

19. Andrade JM, Faustino C, Garcia C, Ladeiras D, Reis CP, Rijo P (2018) Rosmarinus officinalis L.: an update review of its phytochemistry and biological activity. Future Sci OA 4(4):FSO283. https://doi.org/10.4155/fsoa-2017-0124

20. Gao Q, Liu H, Yao Y, Geng L, Zhang X, Jiang L, Shi B, Yang F (2015) Carnosic acid induces autophagic cell death through inhibition of the Akt/mTOR pathway in human hepatoma cells. $\mathrm{J}$ Appl Toxicol 35(5):485-492. https://doi.org/10.1002/jat.3049

21. Park JE, Park B, Chae IG, Kim DH, Kundu J, Kundu JK, Chun KS (2016) Carnosic acid induces apoptosis through inactivation of Src/STAT3 signaling pathway in human renal carcinoma Caki cells. Oncol Rep 35(5):2723-2732. https://doi.org/10.3892/or. 2016.4642

22. Kim DH, Park KW, Chae IG, Kundu J, Kim EH, Kundu JK, Chun KS (2016) Carnosic acid inhibits STAT3 signaling and induces apoptosis through generation of ROS in human colon cancer HCT116 cells. Mol Carcinog 55(6):1096-1110. https://doi.org/ $10.1002 / \mathrm{mc} .22353$

23. Gonzalez-Vallinas M, Molina S, Vicente G, Zarza V, MartinHernandez R, Garcia-Risco MR, Fornari T, Reglero G, Ramirez de Molina A (2014) Expression of microRNA-15b and the glycosyltransferase GCNT3 correlates with antitumor efficacy of Rosemary diterpenes in colon and pancreatic cancer. PLoS ONE 9(6):e98556. https://doi.org/10.1371/journal.pone.0098556

24. Kar S, Palit S, Ball WB, Das PK (2012) Carnosic acid modulates $\mathrm{Akt} / \mathrm{IKK} / \mathrm{NF}-\mathrm{kappaB}$ signaling by PP2A and induces intrinsic and extrinsic pathway mediated apoptosis in human prostate carcinoma PC-3 cells. Apoptosis 17(7):735-747. https://doi.org/10. 1007/s10495-012-0715-4

25. Han NN, Zhou Q, Huang Q, Liu KJ (2017) Carnosic acid cooperates with tamoxifen to induce apoptosis associated with Caspase-3 activation in breast cancer cells in vitro and in vivo. Biomed Pharmacother 89:827-837. https://doi.org/10.1016/j.biopha.2017.01. 084

26. Yildiz-Ozturk E, Gulce-Iz S, Anil M, Yesil-Celiktas O (2017) Cytotoxic responses of carnosic acid and doxorubicin on breast cancer cells in butterfly-shaped microchips in comparison to $2 \mathrm{D}$ and 3D culture. Cytotechnology 69(2):337-347. https://doi.org/ 10.1007/s10616-016-0062-3

27. Wang LQ, Wang R, Li XX, Yu XN, Chen XL, Li H (2015) The anti-leukemic effect of carnosic acid combined with adriamycin in a K562/A02/SCID leukemia mouse model. Int J Clin Exp Med 8(7):11708-11717

28. Wang R, Cong WH, Guo G, Li XX, Chen XL, Yu XN, Li H (2012) Synergism between carnosic acid and arsenic trioxide on induction of acute myeloid leukemia cell apoptosis is associated with modulation of PTEN/Akt signaling pathway. Chin J Integr Med 18(12):934-941. https://doi.org/10.1007/s11655-012-1297-z

29. Marino G, Niso-Santano M, Baehrecke EH, Kroemer G (2014) Self-consumption: the interplay of autophagy and apoptosis. Nat Rev Mol Cell Biol 15(2):81-94. https://doi.org/10.1038/nrm3735 
30. Lockshin RA, Zakeri Z (2004) Apoptosis, autophagy, and more. Int J Biochem Cell Biol 36(12):2405-2419. https://doi.org/10. 1016/j.biocel.2004.04.011

31. Teocchi MA, D’Souza-Li L Apoptosis through death receptors in temporal lobe epilepsy-associated hippocampal sclerosis. (1466-1861 (Electronic)). doi:D - NLM: PMC4781997 EDAT2016/03/24 06:00 MHDA- 2016/12/31 06:00 CRDT- 2016/03/24 06:00 PHST- 2015/09/24 00:00 [received] PHST- 2016/01/05 00:00 [revised] PHST- 2016/01/10 00:00 [accepted] PHST2016/03/24 06:00 [entrez] PHST- 2016/03/24 06:00 [pubmed] PHST- 2016/12/31 06:00 [medline] AID - 10.1155/2016/8290562 [doi] PST - ppublish

32. Chowdhury I, Tharakan B, Bhat GK (2008) Caspases - an update. Comparative biochemistry and physiology Part B. Biochem Mol Biol 151(1):10-27. https://doi.org/10.1016/j.cbpb.2008.05.010

33. Boulares AH, Yakovlev AG, Ivanova V, Stoica BA, Wang G, Iyer S, Smulson M (1999) Role of poly(ADP-ribose) polymerase (PARP) cleavage in apoptosis caspase 3-resistant parp mutant increases rates of apoptosis in transfected cells. J Biol Chem 274(33):22932-22940
34. Mizushima N, Komatsu M (2011) Autophagy: renovation of cells and tissues. Cell 147(4):728-741. https://doi.org/10.1016/j.cell. 2011.10.026

35. Tanida I, Ueno T, Kominami E (2004) LC3 conjugation system in mammalian autophagy. Int J Biochem Cell Biol 36(12):25032518. https://doi.org/10.1016/j.biocel.2004.05.009

36. Katsuragi Y, Ichimura Y, Komatsu M (2015) p62/SQSTM1 functions as a signaling hub and an autophagy adaptor. FEBS J 282(24):4672-4678. https://doi.org/10.1111/febs.13540

37. Li H, Jin X, Zhang Z, Xing Y, Kong X (2013) Inhibition of autophagy enhances apoptosis induced by the PI3K/AKT/mTor inhibitor NVP-BEZ235 in renal cell carcinoma cells. Cell Biochem Funct 31(5):427-433. https://doi.org/10.1002/cbf.2917

38. Ouyang L, Shi Z, Zhao S, Wang FT, Zhou TT, Liu B, Bao JK (2012) Programmed cell death pathways in cancer: a review of apoptosis, autophagy and programmed necrosis. Cell Prolif 45(6):487-498. https://doi.org/10.1111/j.1365-2184.2012.00845.x 